\title{
Prenatal sFlt-1/PIGF value for prediction of adverse neonatal outcome of isolated ultrasonic small-for- gestational-age fetus in normotensive pregnant women: A prospective cohort study
}

\section{So Hyun Shim}

CHA Gangnam Medical Center

Haeng Jun Jeon

CHA Gangnam Medical Center

Hye Jin Ryu

CHA Gangnam Medical Center

So Hyun Kim

CHA Gangnam Medical Center

\section{Seung Gi Min}

CHA Gangnam Medical Center

\section{Min Kyu Kang}

CHA Gangnam Medical Center

Hee Jin Park

CHA Gangnam Medical Center

Dong Hyun Cha ( $\nabla$ chadh001@chamc.co.kr)

CHA Gangnam Medical Center

\section{Research article}

Keywords: Soluble fms-like tyrosine kinase-1, Placental growth factor , Small for gestational age, Fetal growth restriction, Biomarker, Normotensive pregnancy

Posted Date: August 4th, 2019

DOI: https://doi.org/10.21203/rs.2.12396/v1

License: (9) (1) This work is licensed under a Creative Commons Attribution 4.0 International License. Read Full License 


\section{Abstract}

Background Not only preeclampsia but also small-for-gestational-age (SGA) neonates in the absence of preeclampsia are at increased risk of morbidity and mortality. Early recognition of fetuses at increased risk of being growth-restricted enables more appropriate surveillance and optimization of management for reduced risk of adverse neonatal outcomes. We investigated potential value of soluble fms-like tyrosine kinase-1 (sFlt-1) to placental growth factor (PIGF) ratio, estimated in late-second and early-third trimester respectively, for prediction of SGA neonates with poor neonatal outcome. Methods Included in this case control study were 530 singleton pregnant women who had attended the prenatal screening program at single institution between January 2011 and March 2012. The maternal serum levels of sFlt-1 and PIGF at 24 to $28+6$ weeks and 29 to $36+6$ weeks of gestation were measured for comparing control and SGA group. Results After excluding 22 preeclampsia cases, 47 SGA group and 461 control-group were included. Mean gestational age at delivery in SGA group was $37.76 \pm 2.05$ weeks, which showed no significant difference comparing to control group (38.43 \pm 2.1 weeks, $p=0.122)$. The sFlt-1/PIGF ratios at late-second trimester and early-third trimester were both higher in the SGA group than control group (7.62 vs $3.74, p=0.63 ; 28.62$ vs $14.42, p=0.037$ ) and especially sFlt-1/PIGF ratio at early-third trimester was significantly higher in the SGA group. Among SGA group, 17 neonates had adverse neonatal outcome $(36.1 \%, 17 / 47)$. Both sFIt-1/PIGF ratios in each trimester were also higher in poor SGA group than control group (6.73 vs $3.77, p=0.379 ; 109.12$ vs $15.27, p=0.002$ ). As gestational age advances, rapid increase in sflt-1/PIGF ratio detected in poor SGA group comparing to SGA group with no adverse outcome. A cutoff value of 28.15 for the sFlt-1/PIGF ratio at 29 to $36+6$ weeks significantly predicted SGA neonates who had adverse outcome, with sensitivity and specificity of $76.9 \%$ and $88 \%$, respectively. Conclusion In this study, sFlt-1/PIGF ratio of SGA with adverse neonatal outcome group was significantly higher than control group. This study suggests the feasibility of the sFIt-1/PIGF ratio as helpful objective measurement for predicting the adverse SGA neonatal outcome by providing sFIt-1/PIGF cutoff value, besides ultrasound biometry measurement.

\section{Background}

Generally, small-for-gestational-age (SGA) fetuses are defined as those having gestational weights below the tenth percentile. During the prenatal period, ultrasound SGA fetuses are detected at a rate of around $5 \sim 8 \%$ of all late-phase pregnancies(1). When SGA fetuses with combined clinically suspected preeclampsia (PE) status are detected on prenatal ultrasonography, they are carefully evaluated for Intrauterine Growth Restriction (IUGR) status, which can lead to fetal death due to pathologic intrauterine fetal asphyxia. With no clinical PE clues or any other abnormal findings of the Doppler index, which is the tool of diagnosis of fetal asphyxia, incidentally detected isolated ultrasonic SGA fetuses have been given normal antenatal care(2-4). Hence these fetuses have long been considered to be constitutionally small babies with a good perinatal outcome. However, as clinical study results have accumulated over the recent decades, it has become clear that SGA fetuses have poorer perinatal outcomes, such as suboptimal neurodevelopment and higher postnatal cardiovascular risk, than do appropriate for 
gestational age (AGA) newborns(2-9). One of those studies reported that impaired fetal growth is a serious complication in pregnancy, being a major determinant of perinatal morbidity and mortality(9).

Although the exact pathophysiologies of poor fetal growth remain unknown, hypoperfusion from incomplete trophoblastic invasion is known to be the most crucial causative factor for SGA. Impaired trophoblast invasion results in decreased feto-placental perfusion and placental dysfunction, which, finally, leads to poor pregnancy outcomes. The placenta of SGA infants shows reduced intervillous space volume and parenchyme, with few chorionic villies. A well-known diagnostic tool of pathologic fetal growth restriction is the UA Doppler index. Increased UA systolic-diastolic ratio is considered to be indicative of poorly vascularized placental villi status, though such status is seen only in extremely progressed cases. With earlier detection of pathologic fetal growth restriction, other Doppler ultrasound indices, such as reduced values of middle cerebral artery (MCA) Doppler or cerebroplacental ratio (CPR), have been clinically applied. However, ultrasound surveillance requires trained personnel and equipment, which may not be readily available for all diagnosed SGA pregnancies(1).

Consequently, a clinical need has emerged to develop additional biochemical markers to improve current diagnostic accuracy and reduce the need for ultrasound-based examinations for detection of SGA fetuses. The most widely studied angiogenic markers are placental growth factor (PIGF) and soluble fmslike tyrosine kinase-1 (sFlt-1). Uteroplacental insufficiency is associated with unbalanced angiogenesis, which results in up-regulation of sFlt-1 and down-regulation of PIGF. So, the sFlt-1/PIGF ratio is significantly higher in cases of uteroplacental insufficiency such as PE originating from placental insufficiency, and has been commercialized and employed in cases of suspected PE to predict poor maternal and perinatal outcomes and help clinicians to determine the most appropriate delivery times.

However, clinicians have to be concerned not only about fetal growth restriction combined with PE but also isolated ultrasonic SGA with no other abnormalities and the optimal management of such cases. Despite the fact that isolated SGA fetuses are also known to be associated with placenta insufficiency, there has been a tendency to focus on mostly SGA with PE and to study only the relevant biomarkers. There has been little research on biomarkers for poor perinatal outcomes in cases of isolated SGA in the absence of PE. As not only PE neonates but also SGA neonates in the absence of PE are at increased risk of morbidity and mortality after birth, a new approach and a different perspective are needed for evaluation of isolated SGA. Early recognition of fetuses at increased risk of being only growth-restricted might enable more appropriate surveillance and (thereby) optimized management, which have been shown to reduce the risk of adverse fetal outcome.

In the present study, we investigated the potential value of maternal serum levels of the sFIt-1/PIGF ratio for prediction of adverse neonatal outcome for isolated SGA fetuses in the absence of PE, and also investigated whether other factors can predict poor perinatal outcomes. Additionally, we endeavored to determine the clinically significant cut-off value of sFIt-1/PIGF for prediction of poor neonatal outcome in cases of isolated ultrasonic SGA fetuses. 


\section{Methods}

Ours was a prospective cohort study that included 530 singleton pregnant women who had attended a prenatal screening program at CHA Gangnam Medical Center in Seoul, Korea, between January 2011 and March 2012. Written consent was obtained from all of the participants, and the study was approved by the Institutional Review Board of CHA Gangnam Medical Center, CHA University. The women were interviewed about their obstetric data and medical history. The gestational age was assessed by embryo fetal crown-rump length in the first trimester. At the gestational ages of 10 weeks and 13 weeks, 6 days, maternal serum levels of pregnancy-associated plasma protein-A (PAPP-A) and fetal nuchal translucency were measured; subsequently, at the gestational ages of 15 weeks and 20 weeks, 6 days, 4 markers (alpha-fetoprotein (AFP), unconjugated estriol (uE3), inhibin-A, and human chorionic gonadotropin (hCG)) were measured as a Down syndrome screening procedure. All of the markers were measured using a UniCel Dxl 800 analyzer (Beckman Coulter Inc., Fullerton, CA, USA), and the values were transformed to multiples of the median (MoM) after adjusting for gestational age and maternal BMI.

We sequentially analyzed 2 periods of gestational age: $24+0$ to $28+6$ weeks of gestation (early phase) and $29+0$ to $36+6$ weeks of gestation (late phase).

We also measured, as additional angiogenic biomarkers, the maternal plasma levels of the sFlt-1 and PIGF values at both early-phase gestation and late-phase gestation. After clotting, the samples were centrifuged, and plasma was stored at $-80^{\circ} \mathrm{C}$. The sFIt -1 and PIGF levels of each of the samples were measured simultaneously using the fully automated Roche Diagnostics Elecsys assay (Roche Diagnostics, Penzberg, Germany), and the sFlt-1/PIGF ratio was calculated.

At each trimester, an ultrasound scan also was performed. Fetal biparietal diameter, femur length, and abdominal and head circumference were measured using the ATL-5000 ultrasound system (Philips Medical Systems, Andover, MA, USA). Uterine-artery (UtA) Doppler ultrasonography with color-flow mapping was performed at the gestational ages 20 weeks and 24 weeks, 6 days. Both left and right UtA blood flows were examined using color Doppler imaging. The Doppler gate was placed at the proximal UtA, according to the Fetal Medicine Foundation guidelines. The pulsatility index (PI), resistance index $(\mathrm{RI})$, and systolic diastolic (S/D) ratio were measured, and the average of the measurements from the left and right UtA was used for the analysis. The Doppler measurements were performed by 3 well-trained examiners.

In our study, the control group was defined as AGA with no concerns regarding PE before or after delivery. The diagnosis of PE was made according to the National High Blood Pressure Education Program Working Group on High Blood Pressure in Pregnancy criteria. Hypertension was defined as repeated systolic blood pressure measurements of $\geq 140 \mathrm{mmHg}$ (Korotkoff phase 1) and diastolic blood pressure measurements of $\geq 90 \mathrm{mmHg}$ (Korotkoff phase 5). Proteinuria was defined as repeated $\geq 1+$ proteinuria on dipstick urinalysis or $\geq 300 \mathrm{mg}$ of protein in a 24-hour urine collection sample. Our SGA group was defined as infant birth weight less than the tenth percentile of the corresponding curves after adjustment for gestational age. 
Adverse neonatal outcomes regarded as requiring NICU admission were attributed to sub-causes including jaundice, meconium aspiration syndrome, transient tachypnea of newborn, respiratory distress syndrome, necrostizing enterocolitis, sepsis, and use of ventilation.

Information on the presence of obstetric complications, pregnancy outcome and fetal outcome, including fetal sex, gestational age at delivery and birth weight, were obtained after delivery.

All of the statistical analyses were performed using SPSS for Windows version 21.0 (SPSS Inc., Chicago, IL, USA) software. All of the values were recorded in means ( \pm SD), and the analysis method entailed use of the Mann-Whitney $U$ test. Due to the fact that the sFIt-1/PIGF repeated-measure analysis of the second and third trimesters in each group did not satisfy the requirements for sphericity of data analysis, a mixed version of the general liner model, an analysis technique similar to repeated-measure ANOVA, was employed. Values of $p<0.05$ were considered statistically significant. Receiver operating characteristic (ROC) curves were constructed using logistic regression analysis. The resulting areas under the ROC curves (AUC) were compared by pairwise analysis.

\section{Results}

Among the total 530 study recruits, 22 (4.2\%) pregnant women were diagnosed as PE. After excluding these 22 PE singletons, 508 sets of pregnancy data were analyzed. Among these, 47 SGA singletons and 461 control-group subjects were enrolled and compared to identify the relationship between each of the markers and the customized birth weight.

The basic characteristics of the 2 subjects are summarized in Table 1. The mean age and age distribution of the 2 groups were similar. The gestational weeks at delivery, sex dispersion of baby, hemoglobin in early pregnancy, the gestational weeks at $1^{\text {st }}, 2^{\text {nd }}$ serum tests and blood pressure in early pregnancy showed no differences between the 2 groups. Pre-pregnancy maternal weight and body-mass index (BMI) were significantly lower in the SGA group ( 54.8 vs. $49.9 \mathrm{~kg}, \mathrm{p}=0.00 ; 20.2 \mathrm{vs} .18 .8 \mathrm{~kg} / \mathrm{m}^{2}, \mathrm{p}=0.00$ ). The percentage of primigravidarum women was as high as $80.9 \%$ in the SGA group.

Table 2 compares the angiogenic factors and Doppler results between the 2 groups. The MoM values of the PAPPA of the $1^{\text {st }}$ trimester screening test and the hCG and uE3 levels of the $2^{\text {nd }}$ trimester screening test were significantly lower in the SGA group ( 1.25 vs. $1.00, p=0.036 ; 1.16$ vs. $0.93, p=0.001 ; 1.09$ vs. $0.99, p=0.041)$. Nuchal translucency of $1^{\text {st }}$ trimester and the AFP and inhibin-A levels of the $2^{\text {nd }}$ trimester showed no difference.

Among the serum levels measured at $24-28+6$ weeks (early-phase gestation), the sFlt- 1 level and sFlt1/PIGF ratio (ratio I) were higher and the PIGF level was lower in the SGA group, but the differences were not statistically significant. The sFIt-1/PIGF ratio as determined at 29-36+6 weeks (late-phase gestation; ratio II), however, was significantly higher in the SGA group (14.42 vs. $28.62, p=0.037)$. As is known, it has been statistically confirmed that neonatal birth weight and sFlt-1/PIGF ratio I, II were statistically were inversely proportional (data not shown). There were also significant differences in the 
uterine-artery Doppler ultrasonography measurements between the 2 groups. All of the 3 Doppler impedance indices were higher in the SGA group than in the control group (RI: 0.55 vs. $0.61, p=<0.01$; Pl: 0.93 vs. $1.13, p=0.02 ; S / D$ ratio: 2.35 vs. $2.85, p=0.009$ ).

Figure 1 plots the ROC curves of sFIt-1/PIGF ratio II for prediction of SGA at birth. The cut-off value was 11.25 with $60.0 \%$ sensitivity and $61.9 \%$ specificity (AUC area: $0.663(95 \% \mathrm{Cl}, 0.564-0.762)$ ).

The neonatal outcome data is also presented in Table 2. Fifty-six (56) neonates were admitted to the $\mathrm{NICU}$, and the admission rate was significantly higher in the SGA group (9.5 vs. $25.5 \%, \mathrm{p}=0.001$ ). Concomitant complications were jaundice, meconium aspiration syndrome, Transient tachypnea of newborn, Respiratory distress syndrome, Necrotizing enterocolitis, sepsis, and hypoxia, all of which were more common in the SGA group.

To determine the clinically applicable cut-off value for prediction of SGA neonates, especially those who showed adverse neonatal outcome, Table 3 shows basic comparison between control and adverse neonatal result group. Among SGA group, 17 neonates had adverse neonatal outcome $(36.1 \%, 17 / 47)$. Both sFIt-1/PIGF ratios in each trimester were also higher in poor SGA group than control group (6.73 vs $3.77, p=0.379 ; 109.12$ vs $15.27, p=0.002$ ). The Figure 2 ROC curve shows a much higher correlation between sFIt-1/PIGF ratio II and prediction of SGA at birth with adverse neonatal outcome among those subsequently admitted for NICU care. The cut-off value of sFIt-1/PIGF ratio II was 28.15 with $76.9 \%$ sensitivity and $88 \%$ specificity (AUC area: 0.907 (95\% Cl, 0.829-0.985)).

\section{Discussion}

This study confirmed that in most cases of SGA, especially in those showing adverse neonatal outcome, circulating levels of sFIt-1 are elevated and those of PIGF are diminished, resulting in an increased sFlt1/PIGF ratio, similarly to what occurs in PE. We demonstrated the possibility of the clinical application of a maternal angiogenic marker (sFlt-1/PIGF) to determine optimal delivery times for minimization of poor neonatal outcome in cases of ultrasonic suspected SGA in the absence of PE.

What should be the next step for clinicians in cases of incidentally detected ultrasonic SGA? Differential diagnosis between fetuses that are small due to 'placental intrauterine growth restriction' and those that are 'constitutionally small fetuses' has been considered a major area of interest in clinical obstetrics(1014). The important preliminary step would be to determine PE coexistence. Not only maternal blood pressure and proteinurea, but also UA Doppler flow is used to determine the risk of adverse neonatal outcome. Most studies agree that abnormal UA Doppler flow can suggest fetuses at higher risk of SGA including PE. But Figueras F et al. pointed out that there is a possibility of UA Doppler false positivity. Indeed, normal antenatal UA Doppler cannot be taken as a single indicator of low adverse-neonataloutcome risk, as SGA with normal umbilical artery also has shown a high prevalence of adverse perinatal outcome (15). Another limitation of UA Doppler is that its findings are affected by individual examiners and thus might be somewhat subjective. 
Consequently, angiogenic biomarkers have become an important focus in obstetrics, especially in the diagnosis of placenta-origin diseases such as PE. Many results have shown that imbalances in angiogenic and anti-angiogenic factors as measured in maternal blood are detectable prior to clinical diagnosis, and that such measurements have high prognostic value (16-20). Uteroplacental insufficiency is associated with unbalanced angiogenesis and resultant up-regulation of sFlt-1 and down-regulation of PIGF. Abnormal levels of angiogenic factors, as reflected in the maternal circulation, play a key pathogenic role in the development of the endothelial dysfunction underlying $\mathrm{PE}$. The value of angiogenic biomarkers in the prediction and characterization of early-onset PE and FGR has been demonstrated in a large number of studies; already, in fact, a diagnostic serum kit based on the sFIt/PIGF ratio is being used in outpatient clinics. Gestation-age-specific sFIt/PIGF ratio cut-offs of $>85(20+0$ to $33+6$ weeks) and $>110$ (34+0 weeks to delivery) have been shown to be highly suggestive of PE. In the PROGNOSIS study, a single sFIt-1/PIGF ratio cut-off (<38) was validated as accurately and reliably ruling out PE within 1 week (negative predictive value $>96 \%$ ) and confirming PE $(>=38)$ within 4 weeks (positive predictive value $>25 \%$ ). As such, it can be considered to be of great value to both clinicians and patients. The rate of incidence of PE in Asian women, however, is relatively lower than in other ethnic groups (e.g., AfricanAmericans), which fact has resulted in a paucity of PE studies on Asian populations. The present study's population, uniquely, was Asian (i.e., Korean) women. Especially for such a country as Korea, in which the incidence of PE is low, our present results suggest an optimal and novel approach that utilizes the sFlt1/PIGF ratio as a highly effective prognostic indicator of adverse outcome in cases of isolated SGA status.

It must be noted, though, that at the third trimester, unlike the first and second, SGA and placental insufficiency might not be noticeable, because SGA determination is made only by ultrasound biometry routinely. Thus, besides ultrasound biometry measurement for prediction of SGA, objective measurements are needed.

This study is the first (as ascertained by a PubMed search of English language papers from 1996 to December 2018 using the search terms 'PIGF' and 'sFIt-1') to examine, among a cohort of SGA fetuses, the utility of angiogenic factors for prediction of adverse outcome in cases where PE is absent. Over the last few years, several reports have provided evidence that angiogenic markers also differ with respect to late-pregnancy complications. Accordingly, PIGF levels are lower in pregnancies with SGA. However, no studies have evaluated angiogenic factors to determine whether they predict poor outcome among SGA fetuses or how they compare with Doppler ultrasound surveillance. In several preceding studies, maternal serum angiogenic factors in SGA-pregnancy women with PE have been scrutinized. One study reported that among patients suspected of having SGA fetuses, abnormal maternal plasma concentrations of angiogenic/anti-angiogenic factors were 5-9 and 8-9 times more likely to lead to PE or preterm delivery(21). Ignacio et al. reported the following median values of sFIt-1/PIGF: control group 11.0, fetal growth restriction group 116.8, PE 66.5, and PE combined with fetal growth restriction group 165.4(22). However, they could not determine the cut-off value. In our study, we demonstrated that the early-sFlt1/PIGF ratio was not remarkable in predicting obstetric outcome but that increased sFIt-1/PIGF ratio checked at 29 to $36+6$ weeks (late-phase gestation) was strongly predictive of adverse neonatal outcome 
with a cut-off value of 28.15 (76.9\% sensitivity and $88 \%$ specificity, AUC area: 0.907$)$. Moreover, increased sFlt-1/PIGF ratio checked at the same time with a cut-off value of 11.25 was shown to be a clinically predictive marker of real SGA neonates in cases of ultrasonic SGA $(60.0 \%$ sensitivity and $61.9 \%$ specificity; AUC area: $0.663 ; 95 \% \mathrm{Cl}, 0.564-0.762$ ) beyond the clinical and ultrasonic values.

The PAPP-A, which is produced by developing trophoblast cells, in the maternal serum is known to decrease in PE populations(23-25). In a multicenter study of 8839 women, a significant relationship between low PAPP-A levels and IUGR, preterm delivery, PE and stillbirth were reported(26). Low levels of PIGF and PAPP-A in the maternal serum may reflect impaired placentation, which results in the development of fetal growth restriction(27). In our study, HCG and E3 levels in SGA group were lower than control group. There is no established association with the HCG and E3 levels in SGA group, but it is thought to be a good indicator for predicting poor fetal growth if more results are built up in the future.

This study has important clinical implications. Effective screening for detection of adverse perinatal outcome in cases of isolated SGA is an area of unmet clinical need. In the study, we serially evaluated not only UtA Doppler but also sFIt-1/PIGF ratio data for cases of suspected SGA, determining that sFlt-/PIGF can be an independent marker of poor neonatal outcome. Such biomarkers can be identified from a simple blood test; thus, this approach can be especially useful in areas where access to Doppler examination expertise is limited. If these results are corroborated by others, the strong potential of angiogenic biomarkers for risk stratification in cases of ultrasonic isolated SGA and subsequent reduction of both morbidity and healthcare costs in the management of SGA fetuses will be confirmed.

\section{Conclusion}

At the third trimester, unlike the first and second, SGA and placental insufficiency might not be noticeable, because SGA determination is made only by ultrasound biometry. Thus, besides ultrasound biometry measurement for prediction of SGA, this study explored the feasibility of the early-third-trimester sFlt/PIGF ratio as an objective measurement and a possibly more useful predictor of SGA and adverse neonatal outcome than maternal Doppler indices.

\section{List Of Abbreviations}

\section{sFlt-1: soluble fms-like tyrosine kinase-1}

\section{PIGF: placental growth factor}

\section{PE: preeclampsia}

\section{IUGR: Intrauterine Growth Restriction}




\section{SGA: small-for-gestational-age}

\section{AGA: appropriate for gestational age}

\section{PAPP-A:pregnancy-associated plasma protein-A}

\section{AFP: alpha-fetoprotein}

\section{uE3: unconjugated estriol}

\section{hCG: human chorionic gonadotropin}

\section{UtA: uterine artery}

\section{MCA: middle cerebral artery}

\section{Declarations}

Consents for publication: Not applicable

Competing interests: The authors declare that we have no competing interests

Funding: This research was funded by the Ministry of Health \& Welfare, Republic of Korea

Authors' contribution: SHS was a major contributor in writing the main texts regarding the maternal sFlt1/PIGF value for prediction of adverse neonatal outcome. HJJ, HJR and SHK analyzed and interpreted the patient data. SGM and MKK contributed materials and analysis tools. HJP and DHC performed biochemical examination of biomarkers regarding the pathologic fetal growth restriction. All authors read and approved the final manuscript.

Acknowledgements: This research was supported by a grant of the Korea Health Technology R\&D Project through the Korea Health Industry Development Institute (KHIDI), funded by the Ministry of Health \& Welfare, Republic of Korea (grant number: HI18C-1839-010019)

\section{References}


1.Figueras F, Gardosi J. Intrauterine growth restriction: new concepts in antenatal surveillance, diagnosis, and management. Am J Obstet Gynecol. 2011;204(4):288-300.

2.Figueras F, Oros D, Cruz-Martinez R, Padilla N, Haernandez-Andrade E, Botet F, et al., editors. Neurobehavior in term, small-for-gestational age infants with normal placental function. peds; 2009.

3.Illa M, Coloma JL, Eixarch E, Meler E, Iraola A, Gardosi J, et al. Growth deficit in term small-forgestational fetuses with normal umbilical artery Doppler is associated with adverse outcome. 2009;37(1):48-52.

4.Savchev S, Figueras F, Cruz-Martinez R, Illa M, Botet F, Gratacos EJUiO, et al. Estimated weight centile as a predictor of perinatal outcome in small-for-gestational-age pregnancies with normal fetal and maternal Doppler indices. 2012;39(3):299-303.

5.Comas M, Crispi F, Cruz-Martinez R, Figueras F, Gratacos EJAjoo, gynecology. Tissue Doppler echocardiographic markers of cardiac dysfunction in small-for-gestational age fetuses. 2011;205(1):57. e1-. e6.

6.Eixarch E, Meler E, Iraola A, Illa M, Crispi F, Hernandez-Andrade E, et al. Neurodevelopmental outcome in 2-year-old infants who were small-for-gestational age term fetuses with cerebral blood flow redistribution. 2008;32(7):894-9.

7.Crispi F, Bijnens B, Figueras F, Bartrons J, Eixarch E, Le Noble F, et al. Fetal growth restriction results in remodeled and less efficient hearts in children. 2010.

8.Crispi F, Figueras F, Cruz-Lemini M, Bartrons J, Bijnens B, Gratacos EJAjoo, et al. Cardiovascular programming in children born small for gestational age and relationship with prenatal signs of severity. 2012;207(2):121. e1-. e9.

9.Barker DJ, Osmond C, Winter P, Margetts B, Simmonds SJJTL. Weight in infancy and death from ischaemic heart disease. 1989;334(8663):577-80.

10.Zhang J, Merialdi M, Platt LD, Kramer MS. Defining normal and abnormal fetal growth: promises and challenges. Am J Obstet Gynecol. 2010;202(6):522-8.

11.Ananth CV, Vintzileos AM. Distinguishing pathological from constitutional small for gestational age births in population-based studies. Early Hum Dev. 2009;85(10):653-8.

12.Xu H, Simonet F, Luo ZC. Optimal birth weight percentile cut-offs in defining small- or large-forgestational-age. Acta Paediatr. 2010;99(4):550-5.

13.Galan HL. Timing delivery of the growth-restricted fetus. Semin Perinatol. 2011;35(5):262-9. 
14.von Beckerath AK, Kollmann M, Rotky-Fast C, Karpf E, Lang U, Klaritsch P. Perinatal complications and long-term neurodevelopmental outcome of infants with intrauterine growth restriction. Am J Obstet Gynecol. 2013;208(2):130 e1-6.

15.Figueras F, Eixarch E, Gratacos E, Gardosi J. Predictiveness of antenatal umbilical artery Doppler for adverse pregnancy outcome in small-for-gestational-age babies according to customised birthweight centiles: population-based study. BJOG. 2008;115(5):590-4.

16.Molvarec A, Szarka A, Walentin S, Szucs E, Nagy B, Rigo J, Jr. Circulating angiogenic factors determined by electrochemiluminescence immunoassay in relation to the clinical features and laboratory parameters in women with pre-eclampsia. Hypertens Res. 2010;33(9):892-8.

17.Garovic VD. The role of angiogenic factors in the prediction and diagnosis of preeclampsia superimposed on chronic hypertension. Hypertension. 2012;59(3):555-7.

18.Ghosh SK, Raheja S, Tuli A, Raghunandan C, Agarwal S. Serum PLGF as a potential biomarker for predicting the onset of preeclampsia. Arch Gynecol Obstet. 2012;285(2):417-22.

19.Hagmann H, Thadhani R, Benzing T, Karumanchi SA, Stepan H. The promise of angiogenic markers for the early diagnosis and prediction of preeclampsia. Clin Chem. 2012;58(5):837-45.

20.Verlohren S, Stepan H, Dechend R. Angiogenic growth factors in the diagnosis and prediction of preeclampsia. Clin Sci (Lond). 2012;122(2):43-52.

21.Chaiworapongsa T, Romero R, Whitten AE, Korzeniewski SJ, Chaemsaithong P, Hernandez-Andrade E, et al. The use of angiogenic biomarkers in maternal blood to identify which SGA fetuses will require a preterm delivery and mothers who will develop pre-eclampsia. J Matern Fetal Neonatal Med.

2016;29(8):1214-28.

22. Herraiz I, Droge LA, Gomez-Montes E, Henrich W, Galindo A, Verlohren S. Characterization of the soluble fms-like tyrosine kinase- 1 to placental growth factor ratio in pregnancies complicated by fetal growth restriction. Obstet Gynecol. 2014;124(2 Pt 1):265-73.

23.Deveci K, Sogut E, Evliyaoglu O, Duras NJJoO, Research G. Pregnancy-associated plasma protein-A and $\mathrm{C}$-reactive protein levels in pre-eclamptic and normotensive pregnant women at third trimester. 2009;35(1):94-8.

24.Bersinger NA, Ødegård RAJAoegS. Second-and third-trimester serum levels of placental proteins in preeclampsia and small-for-gestational age pregnancies. 2004;83(1):37-45.

25.Bersinger NA, Smárason AK, Muttukrishna S, Groome NP, Redman CWJHip. Women with preeclampsia have increased serum levels of pregnancy-associated plasma protein A (PAPP-A), inhibin A, activin A and soluble E-selectin. 2003;22(1):45-55. 
26.Smith GC, Stenhouse EJ, Crossley JA, Aitken DA, Cameron AD, Connor JMJTJoCE, et al. Early pregnancy levels of pregnancy-associated plasma protein a and the risk of intrauterine growth restriction, premature birth, preeclampsia, and stillbirth. 2002;87(4):1762-7.

27.Poon L, Akolekar R, Lachmann R, Beta J, Nicolaides KJUiO, Gynecology. Hypertensive disorders in pregnancy: screening by biophysical and biochemical markers at 11-13 weeks. 2010;35(6):662-70.

\section{Tables}

Due to technical limitations, the Table(s) are only available as a download in the supplemental files section.

\section{Figures}




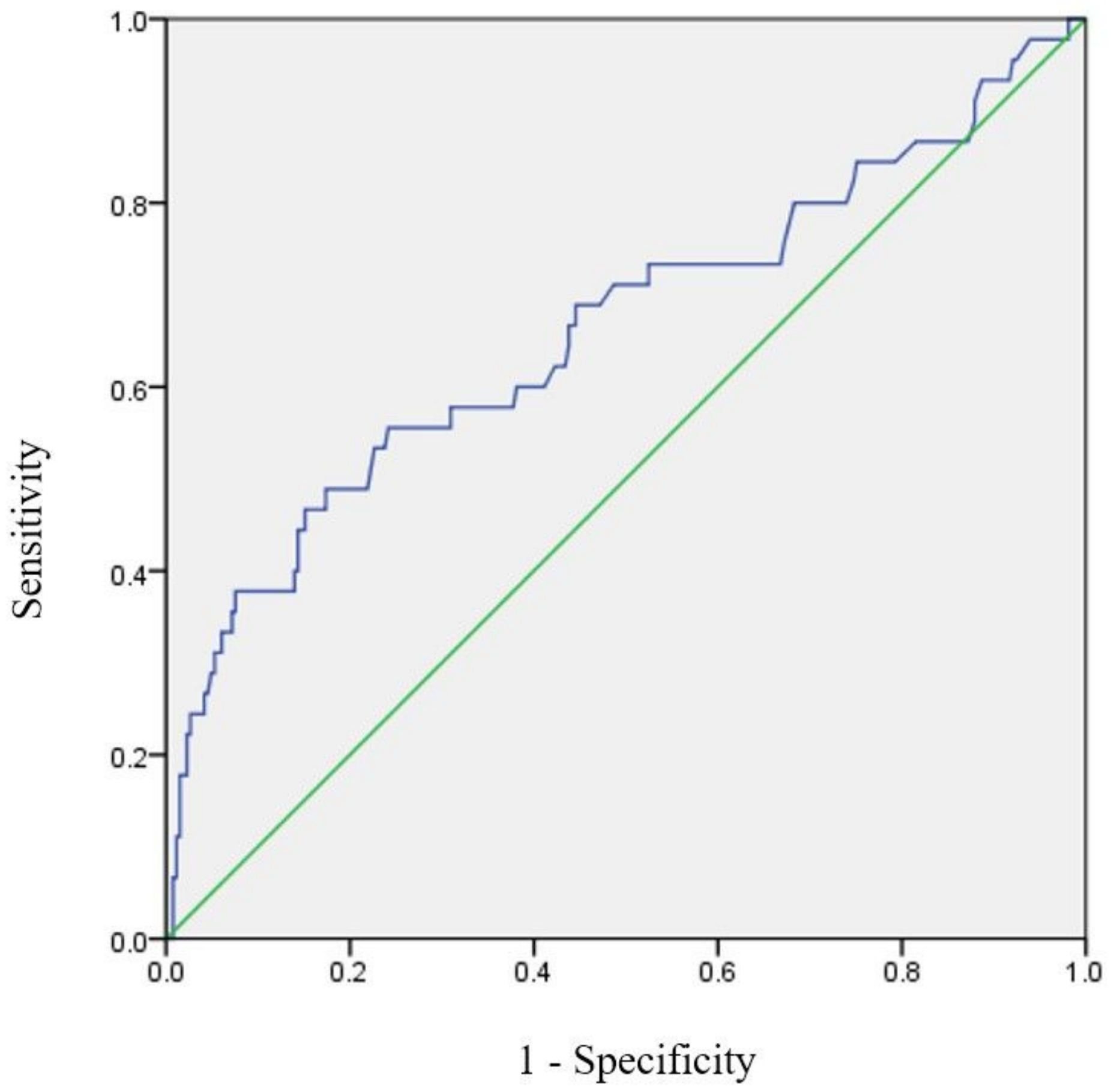

Figure 1

Receiver-operating characteristics curves for prediction of small-for-gestational-age neonates in 508 pregnant women (461 sets of control - group and 47 small-for gestational-age group) by sFlt-1/PIGF ratio at 29 to $36+6$ weeks of gestation. 


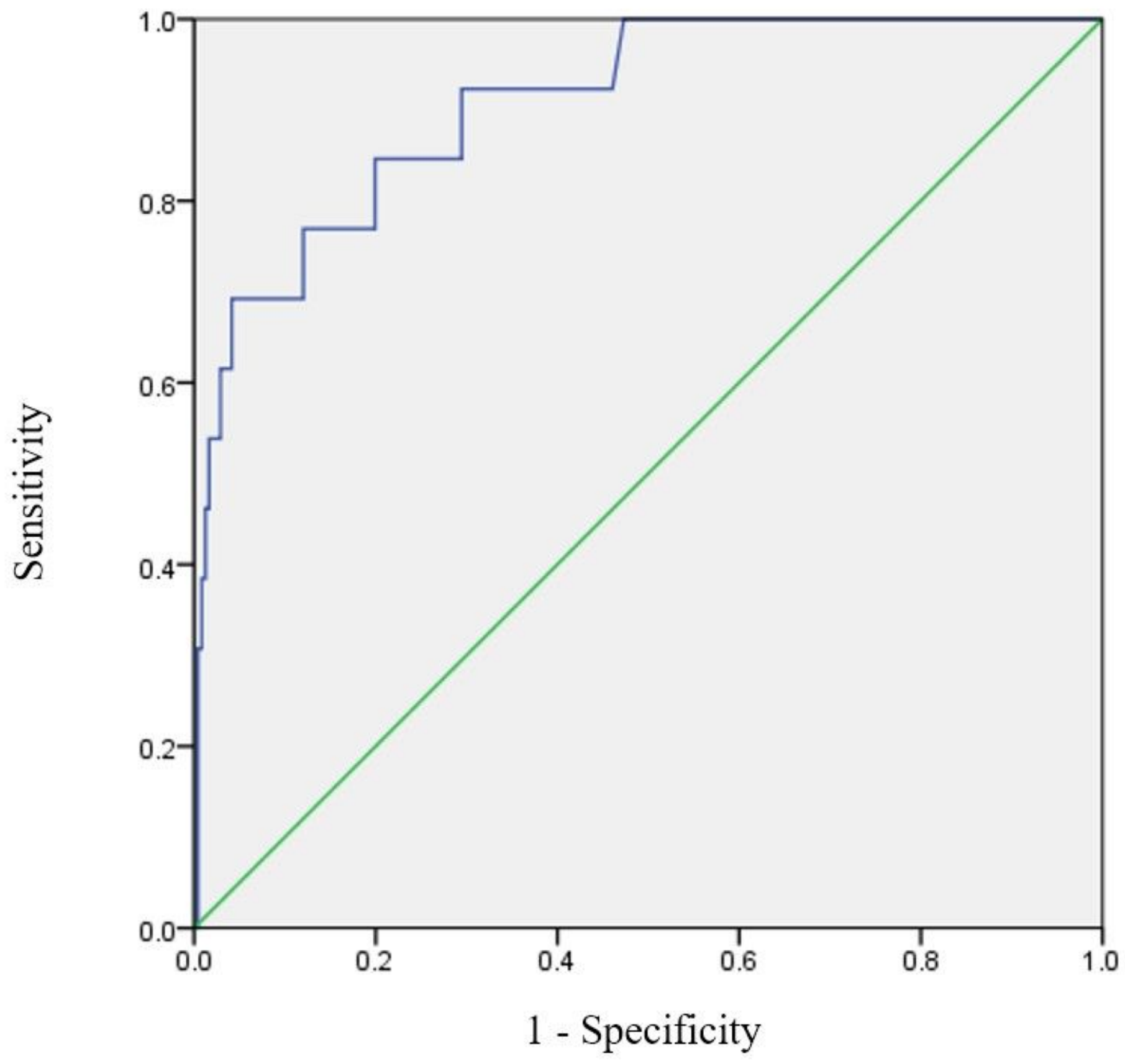

Figure 2

Receiver-operating characteristics curves for prediction of small-for-gestational-age neonates wit adverse neonatal outcome in 478 pregnancy woment (461 sets of control - group and 17 adverse neonatal group) by sFIt-1/PIGF ratio at 29 to $36+6$ weeks of gestation.

\section{Supplementary Files}

This is a list of supplementary files associated with this preprint. Click to download. 
- supplement1.xlsx

- supplement2.xlsx

- supplement2.xlsx 